\title{
Jürgen Habermas Der Philosoph als wahrer Rechtslehrer: Rudolf Wiethölter
}

I.

Als nach beinahe 20 Jahren das legendär gewordene Funkkolleg "Rechtswissenschafu wieder aufgelegt wurde, schrieb Wiethölter ein charakteristisches Vorwort, das den größer gewordenen Abstand zum Texr, gleichzeiug auch die Treve zu den alten Reformhoffnungen zum Ausdruck brachte: „Die politische Ordnung eincr Wirtschaftsgesellschaft ais Rechtskulturverfassung ist und bleibt das theoretische wie praktische Kernstück cines Projekts der Moderne. ' Wie in einem Morto ballen sich die substantivisch zusammengezogenen Gedanken zu Sprengkörpern, die im nächsten Augenblick als ironisches Feuerwerk zerplaczen, um eine allegorische, der Enträtselung bedürfcige Figur an den nächclichen Himmel zu werfen. Es geht Wiethölter um die politische Zährnung und demokratische Verwandlung der kapitaliscischen Gesellschaft im Medium eines Reches, dessen zivilisierende Krafi die kulturellen Lebensformen durchdringt und prägt. Das richtige Recht soll die antagonistische Gescllschaft zur Strcitkultur befreien.

Wiethölter ist cin großer Formulicrer, cin Wortwerker, ein zitatenreicher Jongleur, ein Feuerwerker und Parodisc, der die Worte gewissermaßen schrill zubereitct, damic sie ihre aufklärenden Dienste tun können. Wie Montagen von Alexander Kluge stürzt Wiethöleers sprunghafte Prosa den Leser in Vervirrung. Jeder Satz bündelt dic Gedanken zum Motto, jeder bader sich in einer Fülle von Konnotationen, jeder destruiert ein Vorverständnis, jeder steht gleich nahe zum Mittelpunkt einer geschichtsphilosophischen Holfnung, die sich dem Jahrgang 1929 tief eingeschrieben hat. Mit einem melancholisch gefärbten und doch lebenslang festgehaltenen Aufatmen des Frühjahrs 1945 bleibt Wiechölter seiner Gencracion verhaftct: „... und nach wie vor dic heimliche Holfnung in die verborgenen Pläne der Natur und den öffentlichen Gebrauch der Vernunft, also in jene gutartig-hintcr)istigc Implementationsdialektik, unter deren Zauberbann und Wünscheirute sich Folgen menschlichen Tuns einstellen, die nicht zugleich die Folgen der Absichren menschlichen Tuns gewesen sind. $\mathrm{x}^{2}$

Aus der biographischen Erfahrung des neuen Anfangs mag sich auch die Mencalität und das verhohlene Pathos erklären, die noch den versponnensten Texten ihre Klarlıeit verleihen. Wiechölter gibr nicht auf; auch wenn er sich zurückziche, schwört er nicht ab. In einer temperamentvollen Geburtstagsrede auk den 60jährigen Ludwig von Friedeburg findec sich eincs der seitenen Bckennenisse: „Die damalige Krituk haben wir heute weder zu rechtfertigen noch zu verraten, sondern

I Rudolf Wicthölter, Rechiswissenschafh, Basel 1986, 7

2 Rudalf Wiethölter, Bemerkungen aus der Recht- und Jorsstenvalt, in: Die Zukuní der Aufkarung, Firn. 1989,38 . 
zeitgenössiscl fortzusetzen; zeitgenössisch meint: differenziertcr, komplexer, solider, also auch behutsamer, in einem ganz buchstäblichen Sinne rücksichtsvoller und vorsichtiger - mit Aussicht auf längerfristige Chancen. « Wiechölier beherrsche das Metier der Geburtstagsrede so meisterhaft, daß ich mit ihm nicht in Konkurrenz treten, auf den 60-jährigen Wiethölrer keine Laudario halten möchre. Scattdessen berichte ich von den Erfalhrungen eines juristischen Laien mit dem Juristen und dem wahren Rechtslehrer Wiechölter. Kant meinte damit den Philosophen, der in der Öffentichkeit dic Rolle des Intellektucllen wahrnimmt; aber auch als ein solcher Philosoph bleibe Wiethölter Jurist.

Dem Nicht-Juristen unter den Philosophen bleibt im allgemeinen eine typische Erfahrung nicht erspart: Die öffentlich-rcchtlichen Diskussioncn, zumal jene staatsrechtlichen, die sich im politischen Kräftefcld der Weimarer Republik darbieten, lassen sich an die grobkörnigere philosophische Begrifflichkcit immerhin anschlicBen, während dic Übersicht schwindet und die Verwirrung wächst, je ciefer man in zivilrechtliche Quisquilien eindringt - und alsbald den Wald vor Bäumen nicht mehr sieht. Allein, Wrethölters komplexen Beiträgen komme ich mit diesem handlichen Schema nicht bei. Man muß ihm nur einmal drei Stunden lang zugchört haben! Auf der einen Seite schmeichelt er sich ins Abstraktionsbedürfnis des Philosophen noch wirkungsvoller ein als die Kollegen aus dem Öffentlichen Recht: Wiechölter reißr Perspekriven auf, gibt großarcige Durchblicke. Auf der anderen Seite peinigt Wierhölter den armen Philosophen noch unbarmherziger als dic ohnehin decailbesessenen Privatrcchtler: Ihn läßr Wicchölter mit seinem großen Kaliber hilflos in Bienenschwärmen von skurrilen Einzelfällen sitzen. Wierhölter seziert atemlos einen Fall nach dem anderen um des kaleidoskopischen Effektes willen - alle Strukturen zerfallen in transizorische Einzclheiten und Kontingenzen. Wiethölter verblüff $t$, indem er Konträres nicht erwa vermittelt, sondern absichtsvoll kontaminierc. Der strukturbildende Durchgrifl durchs historische Dickicht wechselt sich ab mit der kasuistischen Zertrümmerung, die das Allgemeine in Besonderen, den Text im Kontext untergehen läßt. Der experimentierenden Geschichtskonstruktion folgt eine Dekonstruktion, die den Virtuosen der Critical Legal Studies zur Ehre gereichen würde. Aber anders als Duncan Kennedy, der sich übrigens in Wiechölters Gedankenwelt bemerkenswert eingefühlt hat, gehört bci diescn beides zusammen : das dekonseruktive Schüren des Krisenbewußrseins und die Suche nach einem konsrruktiven Ausweg.

Wiethölter ist zutiefst davon überzeugt, daß der sozialstaatliche Kompromiß das Rechtssystem entwickelter Industriegesellschaften in eine Krise gestürzt har. Dieses Krisenbewußtsein teilt Wiethölter mit viclen Kollegen; aber nach seiner Autfassung manifestiere sich darin mehr als nur die Überforderung des Rechussystems. Vielmehr sollen dic ungelösten Probleme der Gesellschaft, dic die einen mehr dem Marke, dic anderen mehr dem Staat anlasten, duf ein Versagen des Rechtssystems zurückgehen. Für Wicrhölter ist das Reche kein Subsystem neben anderen, sondern ein ausgezeichnetes Medium der gesamtgesellschaftlichen Inregration: "In der Quintessenz ise von Bedeutung: Marke- und Politikversagen als Rechtsversagen.« Wegen dieser zentralen Stellung soll der Transformarion des Rechessystems und jener rechtstheoretischen Erneucrung, die dazu die Initialzündung gibc, cine cminente politischc Bedeutung zukommen.

Diese beiden Annahmen - über den kritischen Zustand des Rechtssystems und über dic Rechrskrise als Ursache der Gesellschaftskrise - erklären Wrechölters Strategie: Einerseirs versuche er an Einzelfällen immer wieder zu demonstrieren, daß die richterliche Entscheidungspraxis unberechenbar wird, weil konsensstiftende aMaßstäbe, Foren und Verfahrena fehlen; andererseits zieht er aus dieser Diagnose nicht 
die üblichen kontextualistischen Konsequenzen, sondern liält am normativen Selbstverständnis des demokratischen Rechtsstaates fest, um damic die verworrene Praxis doch noch in Einklang zu bringen. Aber sind die Grundannahmen selbst plausibel? Whe müssen wir die These von einer Rechtskrise (II), wie die These von der gesellschaftlich zentralen Sccllung des Rechts (III) verstehen? Ich werde mich auf einigc excgetische Srichworte und Interpolationen beschränken.

$I I$.

Juristen nehmen als geschulce Dogmatiker das Rechr aus der Perspekrive des Richters wahr, und jede Krisc des Rechts lesen sie an der Krise der Rechisprechung ab. Unter diesem Aspekt ist wiederum die Unbestimmtheit der richterlichon Encscheidungen das, was sie am meisten beunruhigt - also der Umstand, daß die Beurteilung eines Falls durch das geltende Recht, durch Präjudizien und Geserzeslage, Kommentare und herrschende Meinung nicht hinreichend determiniert wird (auch wenn sie aufgrund anderer Variablen voraussagbar bleibr). Dabei interessieren vor allem dic strukturellen Gründe: Die incerne Unbestimmtheit der Rechesprechung ergibt sich allgemein aus der Logik der Anwendung von Gesetzesnormen und speziell aus der rechtsförmigen Durchsetzung interventionsstaatlicher Poliriken. Dic wachsende Komplexität der regelungsbedürfrigen Materien und die steigende Interdependenz der Rcgeln selber treiben die Gerichte in das Dilemma, Recht trocz rechtsstaatlicher Gesetzesbindung aus eigener Autorität forbilden zu müssen. Aus dieser Sicht kann von einer Krise des Rechts die Rede sein, wenn sich (a) eine wachsende interne Unbestimmtheit der Rechisprechung diagnoscizieren läßr, die sich (b) als rechclicher Reflex unumkehrbarer policischer und gescllschafelicher Entwicklungen darscellt und (c) vom Richter eine konstruktive, rechtsfortbildende Interpretation verlangt, ohne $d a ß(\delta)$ fïr diese „Abwägung intersubjektiv nachprüfbare und demokratisch legitimierte Maßstäbe (boww. Verlahren der Maßstabsbildung) zur Verfügung stchen: Wer wie Wiethöltcr die Krise des Rechts argwöhnisch aus der Perspektive des Richters verfolgc, muß sich deshalb für das Thema der Rechtsfortbildung interessieren.

In ciner einschlägigen Arbeit räumt Wiethölter gründlich mit der konventionellen Lehre von der Gcwaltentcilung auf. Die Analyse der Rechtsprechung zeige, daß die richterliche Enischeidungspraxis nicht wic im Lchrbuch als ein vergangenheitsorientiertes, nämlich an's bestehende Reche fixiertes Handeln begriffen und von Gesetzgebung und Verwaltung als den auf Zukunft und Gegenwart konzentrierten Handlungsweisen uncerschieden werden kann. Andererseits führt die Liceratur, die Wherhölrer an dieser Stelle erörterr - Studien von F. Müller, D. Simon und R. Ogorek - zu einem ernüchternden, das Krisenszenario durchaus entdramacisierenden Ergebnis. Danach hat sich die Interpretationsarbeit des Richters und der Rechisdogmatik seit dem Ende des 18. Jahrhundercs immer schon innerhalb eines relativ großen Ermessensspielraums bewegt; die richterliche Entscheidungspraxis war ganz unabhängig vom wechselnden politischen und rechtsmerhologischen Rechisverständnis immer schon eine konstruktive Rechtsfortbildung: "Lctzelich sind dem Richcer alle Aufgabenscliritte der regentschaftlichen Vollgewalt geblieben: Er identifiziert den Fall, komponiert das Regelprogramm, trifft Entscheidungen und Verwaltungsmaßnahmen und kalkuliert politische Folgen - und zwar völlig unabhängig davon, ob ihm dies in den lerzten 200 Jahren explizic verboren war, oder ob er 
nachlıaltig dazu ermuntert wurde“s. In scheinbarer Übercinscimmung mic dieser These zieht Wiechölter selbst aus der rechtshiswrischen Untersuchung von R. Ogorek den Schluß, daß sich das Dilemma einer auf Vernunft verpflichteren Jurisprudenz, dic posicives Recht als richtiges Recht nur verwalten kann, indem sie es ohne vermunfurechrliche Rückendeckung und demokratische Legitimation fortbilder, seit dem frühen 19. Jh. kaum verändert haben dürfe.

Wenn aber keine dramatische Zuspitzung jencs Dilemmas, nicht einmal der Trend eines wachsenden Problemdrucks zu erkennen ist, was rechefertigt dann noch die Krisendiagnose, an der Wieshölter auch im Tenor dieser Abhandlung festhält? Was recheferigı die Klage über fehlende »Maßstäbe, Foren und Verfahrena? Warum hält Wiechölter eine tiefgreifende Revision der Grundbegriffe des Rcchts für nörig, um die Krise zu lösen? Warum erhoff́ er sich für cine solche Rekonzcptualisierung des Rechts Hilfe von außen - von systemrheorecischen, von ökonomischen und kommunikationscheoretischen Ansätzen, rivalisierenden Gescllschafestheorien also, dic über die Jnnenperspektive des Rechtssystems hinausgreifen? Wiethölter hält an der altmodischen Anschauung fest, daß das Recht seinen Anspruch auf Legitimität verwirkt, wenn der normative Geltungsanspruch des Rechts nicht mehr durch eine vernünf́tige Jurisprudenz, die die Lücken der Unbestimmtheit schließt, eingelöst werden kann. Er lokalisiert aber dieses Problem nichı auf der Ebene von Methodologie und Rechrsdogmarik unmittelbar, sondern auf der Ebene eines in die Profession eindringenden gesellschafistheoretischen Hintergnundverständnisses, das dem Recht erst einen präzisen Stellenwert gibt, und zwar im Rahmen cines reflexiv gewordenen, dynamisierten Rechtsstaatprojektes: »also muß sich unser Interesse auf die epochalen Einflußoricntierungen richren $\alpha^{4}$.

Das erklärt Wiechölters Inceresse an den "Epochen “ des bürgerlichen Formalrechts, des sozialstaatlich materialisierten Rechts und des prozeduralen Rechts, das einer in Entstchung begriffenen poscindustriellen Gescllschaft angehören soll. Nun gibt diese Periodisienung, wic wir inzwischen wissen, für die tarsächliche Rechtsentwicklung in Gesellschaften unseres Typs nicht allzuviel her. Aber Wiethölter verwendet die Ausdrücke formales, materiales und prozedurales Recht auch nicht für historische Zwecke, sondern als Scichworte für "Sozialmodelle des Rechtsu. Um jene Begriffe kristallisien sich nämlich cin jeweils anderes Hintergrundverständnis derer, die am Prozcß der Verwirklichung des demokratischen Rechtsstaats als cines geschichclichen Projektes teilnehmen - vor allem das der juristischen Experten. Erst der Kontext eines solchen Projekses der gerechien oder wohlgeordneten Gesellschaft (Rarvis) kann nämlich Lücken der Unbestimmtheic einer fallbezogenen Interprecation des geltenden Rechts schlicßen.

Die Rolle von Rechtsideologien, die einen Einbertungskontext für Rcchrsnormen bieten, hat Klaus Günrher jüngst aus der Sicht einer Logik der juristischen Argumentation erläutert. Er hat ein Kohärenzmodell ausgearbeitet, das auf das paradigmatische Hintergrundverständnis von Recht übcrhaupt ein neucs Licht wirft. Die Interpretarion ciner auf Situationen anwendbaren Norm hat das Ziel, den Vorrang der einzig angemessenen Norn so zu begründen, daß das Ideal eincs kohärenten Systems gültiger Normen nicht verletzı wird.' Diejenige Norm, die im Lichtc eincr möglichst vollständigen Beschreibung aller relevanten Merkmalc einer Anwendungssituation als die sangemessene erscheint, setze die Gültigkeit der konkurrierenden, für eine Anwendung zunächst kandidierenden, dann aber als unangemessen

3 D. Simon, zuten nach R. Wietholter, Zum Fortbildungsrecht der ruchterlichen Rexlusfortbildung, KrıV 1988, ilí, 19.

4 Ebenda. Serte 24.

f K. Günther, Der Sinn für Angemessenhert, Fim. 1988. 
zurücktretenden Normen nicht etwa außer Kraft; die Wahl der angemessenen Norm stellt sich vielmehr als Ergebnis der jeweils besten Thcorie aller gültigen Normen dar. Die Interpretation eines Falkes im Lichte der vorrangigen Norm erscheint dann nich ${ }^{6}$ als dic optimalc Verwirklichung eines Rechrsgutes, das mit anderen Rechesgütern konkurricrt; sie bedeutet vielmehr eine unter Berücksichrigung aller Umstände optimale Ausschöpfung eines Systems güluiger Normen.

jede Norm bleibc auf dic kohärente Ergänzung durch alie anderen gültigen Normen angewiescn. Frcilich befindet sich dieses System in ständiger Bewegung, da sich mir jeder neu auftretenden Situation die Vorrangrelationen ändern können. Einc Orientierung an cinem derarr anspruchsvollen Ideal ist in der Regel nicht möglich - und dies nicht einmal im Fall der professionalisierten Rechtsprechung. Anstclle des Ideals treten deshalb Paradigmen, nin dencn Normen, die wir hier und jetzt für gültig halten, (schon) in eine transitjve Ordnung gebracht worden sind... Sie formicren einen Hintergrundkontext, in denen unsere jeweiligen Situationseinschätzungen und die entsprechenden moralischen prima facic-Urteile cingebette sind... So hat unter den modernen Lebensformen jede auf ihre Weise das Rangverhälnis zwischen den Prinzipien der Freiheit und Gleichbeit gelöst, ohne daß man behaupren würde, die entsprechenden Paradigmen seien unveränderlich und würden für jeden Kollisionsfall stets nur ein und dieselbe Lösung vorschieben . Günther fügt aber sogleich hinzu: „Indem sic die Teilnehmer einer besummten gesellschaftlichen Praxis davon entlasten, sich mit einer ungeordncten und nur prima facie anwendbaren Menge von Prinzipien zu begrügen, ... sind die Paradigmen Ireilich auch eine Quelle von Vorurceilen, verzerrten Situationseinschäczungen und entsprechend parreilichen oder einseitigen Anwendungen gülriger Normen. « ${ }^{7}$ Ein Bcispiel dafür bieten die liberalen und die wohlfahrtsstaaclichen Rcchtsideologien mit ihren Kembegriffen des formalen und des materialisierten Rechts. Wiethölters Krisendiagnose läßt sich dann so verstehen, daß diese Paradigmen, die sich um die subjektiven Rechte des privaten Marktteilnehmers und um die Leistungsansprüche der Klienten wohlfahrssstazslicher Bürokratien herum kriscallisiert haben, zerfallen sind, ohne daß eme neue Rechtsideologie an deren Stelle getresen ware. Dic Sozialmodelle, die in den Begriffen des formalen und des materialisierten Rechts impliziert sind, sind heute nicht mehr konsensfähig; sie haben ihre Integrationskraft verloren. Nach dieser Diagnose wird die Rcchtsprechung wunbescimmt", weil der Hintergrundkonsens fehlt, der als Paradigma einer gerechren Ordnung fungieren könnte. Als Altcrnative bietet sich ein funktionalistisches oder ökonomisches Selbstverständnis des Rechtssystems an, das alle normativen Überlegungen deflationiert. Dengcgenüber geht Wiechölter davon aus, daß cin Rechtssystem, welches scine eigenen normativen Grundlagen dcrart dementieren und sich als ein vernunfiloses System von Verhaltenskontrollen darstellen würde, kaum überlebensfähig ist. Er sucht deshalb nach ciner anderen Alternative. Unter dem Stichwort des nprozcduralisierten « Rechts verficht cr das Reflexivwerden aller Paradigmen. Jedenfalis verbinder er mit diesem Begriff das Modell einer sich selbst organisierenden Gesellschaft, die den Pluralismus der Wertorientierungen und Glaubensmächte zur Prämisse has.

An die Stelle idealer produktivistischer Lebensformen, die aus dem Kontrakt freier und gleicher Privatleute oder aus der egalitären Verteilung des gesellschaftsproduzicrten Reichtums hervorgehen, tritt das Projekt der Einrichtung von Verfaltren

6 Wie noch bei R. Alexy. Theoric der Grundicchis, Baden Baden 1985, 71 ff.

7 K. Günther, Ein normariver Begriff der Kohirenz fir eise Theorie der jursstischen Argumentatuon, MS 1989, 22. 
vernünftigen kollektiven Willensbildungsprozessen auf ganzer Breite. An die Stelle einer Zivilreligion dieser oder jencr Provenienz soll cine "Streirkultur des Rechts" treten, an die Stelle eines substantiellen Einbettungskontextes ein solcher, der nicht mehr eine konkrete Lebensform entwirft, sondern nur noch die Verfahren einer Selbstorganisation der Gesellschaft bestimmt.

III.

Wiethölter dekliniert unermüdlich die grammatischen Formen des Rechrs durch. Er uncerscheidet Recht als Formkategorie und Recht als Inhaltskategorie, Recht als Konditional- und als Zweckprogramm, als Form subjektiver Frciheit und als Medium staatlicher Politik in der Absicht, dem zu ergänzenden Tertium in der Reihe der Rechrsformen, der Prozeduralisierung des Rechts, visionäre Konturen zu verleihen. Mit diesem neuen und abstrakten Einbectungskontext soll sich die Rechtskategorie als solche verändern: „Prozeduralisierung zielt nicht so sehr auf soziale Gewährleistungen (als Freiheits-Recht), auch nicht auf Gewährungen (als politische Verwaltung), sondern auf dic Bedingungen der Möglichkeiten ... solcher Gewährleistungen und Gewährungen von integrierbaren Interessenabstimmungen durch rechtliche Verhaltensordnungen. ${ }^{8}$ Wiechölter antizipiert die Einrichtung von Foren und Verfahren für cine diskursive (nach sachlichen und sozialen Bereichen spezifizierte) Willensbildung über Maßstäbe, nach denen die Menge der in unbestimmten Rechtsbegriffen verschlüsselten Prinzipien - wie Kindeswohl, Unternehmensinteresse, Betriebsfrieden, Sozialparität usw. - fallbezogen in eine kohärence Ordnung gebracht werden können. Gleichzeitig dramatisiert er diese Empfelilung zu ciner Perspektive der gesamtgesellschafclichen Transformation: "Prozeduralisicrung ist - in einem Satz - die erneutc Wiederanknüplung an bürgerliche Philosophic-Geschichte in der Absicht, ihre idealistischen wie materialistischen Durchgänge unter verầnderten Umstanden als gesellschaftliclie Lernprojekte zu reproduzieren ${ }^{9}$. An solchen Stcllen schcint Wiethölter zu vergessen, daß zunächst nur von einem prozeduralistischen Hintergrundverständnis die Rede ist, das nach dem Ende aller konsensfähigen Rcchtsideologien dazu dient, die Rationalität der Rechtsprechung zu verbessern. Wierhölter steht erwas Größeres vor Augen. Er hat die vernunftrechtliche Idee der bürgerlichen Gesellschaft als cjncs "eingerichteten und ausgeübtcn Rechtsprogramms noch nicht aufgegeben; er ist nach wie vor davon überzeugt: „Recht (isr) die entscheidende Scruktur der Gesellschaft. «

Das mag aus der Perspektive des Rechtssystcms so erschemen. Aber auch Wiethölter weiß, daß moderne Gesellschaften den Begriffen des rationalen Vernunftrechts und damic den juristischen Grund̈begriffen überhaupt entwachsen sind. Schon die schotrischen Moralphilosophen und die französischen Physiokraten hatten ihre Modelle anderen Wissenschaften entlehnt. Die Gesellschaft wird nicht mehr aus dem Rechr, sondern - von Marx bis Luhmann - das Recht wird aus der Gesellschaft begriffen. In welchem Sinne können wir dann aber von einer zentralen Stellung dos Reches sprechen?

Wiethölter scheint mit Savigny von einem Doppelkonzept des Rechts auszugehen, von Recht als einem funktional spezifizierten Teilbereich und von Recht als einem 
Element, in dem sich das Ganze der Gesellschaft reproduziert. So jedenfalls zitiert er Savigny: „Bey steigender Cultur nämlich sondern sicl alle Thäcigkeiten des Volkes immer mehr, und was sonst gemeinschaftlich becricben wurde, fällt jeczt cinzelnen Ständen anheim. Als ein solcher abgesonderter Stand erscheinen nunmehr auch die Juristen... Das Daseyn des Reches ist von nun an künstlicher und verwickelter, indem es ein doppeltes Leben hat, einnal als Theil des ganzen Volkslebens, was es zu seyn nicht aufhört, dann als besondere Wissenschaft in den Händen der Juristen. Aus dem Zusammenwirken dieses doppelten Lebensprincips crklärcn sich alle spätere Erscheinungen, und es ist nunmehr begreiflich, wie auch jenes ungeheure Derail ganz auf organische Weise, ohne eigentliche Willkühr und Absicht, entszehen konnte. Der Kürze wegen nennen wir künftig den Zusammenhang des Rechts mit dem allgemeinen Volksleben das politische Element, das abgesonderte wissenschaftliche Leben des Rechts abcr das tecbnische Element desselben. « Diese Unterscheidung hat auch heute noch ilıren guren Sinn; das Recht als System aller rechtlich geregelten Interaktionen ist umfassender als das System aller auf Recht reflexiv bezogenen Interaktionen, in denen es um das Recht selbst geht. Aber diescs Rechtssystem im engeren Sinne besteht nicht nur aus der professionellen Anwendung des Rechrs, sondern ebenso aus der Erzeugung und Fortbildung wic der Implementierung von Rechtsnormen oder Rechesprogrammen. Die Innenperspektive dieses Rechtssystems darif nicht einseitig auf die Perspektive des Richters und des Rechtsdogmatikers zurückgeschnitten werden. Wenn eine Perspektive - auch methodisch - in Führung gehen soll, isc es die des demokratischen Gesctzgebers und nicht die des Juristcn, in dem noch Savigny den geborencn Hüter des Rechts und seiner Rationalität gesehen hatıc. Wicthölter richtet seinen Blick gewiß auf das "politische Element " des Rechts - aber auch er privilegiert unversehens dessen wtechnisches Element 4 , sobald es darum geht, wic das Rechtssystem im ganzen auf „Prozeduralisierung urngestellt werden kann. Einige Formulierungen erwecken den Eindnuck, als träte der wahre Rechislehrer noch in der Rolle des Savigny'schen Zivilrechtsdogmatikers auf. Der trug das unsichtbare Gewand des Richterkönigs und stand so hoch, daß er von oben auf den Gesetzgeber als eine seiner Rechrsquellen herabsehen konnte. Alles war dann eine Frage der Klughcir der Jurisprudenz, die die Gesellschaft mit dem richtigen Recht auszustatten hatse - crst cinmal richrig eingerichtet, würde sie schon funktionieren. Ob freilich das Recht in diesem Sinne noch eine zentrale Stelle behaupret, mag man heute in Zweifel ziehen.

In einem anderen Sinne spielt es viellcicht immer noch eine zentrale Rolle - als Statthalter des Anspruchs, daß sich auch unsere komplexen, über Geld und administrative Maclu gesteuerten Systeme nicht vollends einer sozialen, durchs gesamigescllschaftliche Bewußtsein vermittelten Integration entziehen möchten. Parsons hat von der rechthichen Institurionalisicrung des Geld- und des Machtmediums gesprochen. In diesem Sinne ist die Vorstellung vom Recht als dem Medium, über das die Systemintegration angeschlossen blcibt an die soziale Integration der Lebensformen, nicht ganz abwegig. Diese Erwartung verdankt das Recht dem Umstand, daß es Interaktionen gleichzeirig von außen beschränkt und aus der Betciligtenperspekive rechtfertigt; auch wenn es sich mit der Legalität des Handelns begnügt, muß es aus moralischen Gründen immerhin befolgt werden können. So hat Kant das Zrvangsrccht bcgriffen. Und immer noch bindet die normative Dimension seiner Gelcung das Reche im ganzen an cine zwanglos insersubjekcive Anerkennung durch dic Rechtsgcnossen. Solange dem postmetaphysischen Recht auch nur ein Schein von Normativität verbleibt, spiegeln sich rechtliche Organisationsformen im Glanz des Versprechens einer Selbstorganisation der Gescllschaft - dic Betciligten müssen 
die rechclichc Organisation ihres Zusammenlcbens so beqrachten können, als sei diese ihrer vernünfrigen intersubjektiven Willensbildung entsprungen.

Ich vermutc, daß Wierhölter dieses Stück Idealismus, der den Hohn der Zyniker auf sich zieht, im Begriff des "prozeduraicn Rechtse aufgehoben hat. Um cine Veränderung zu bewirken, müßte freilich das radikaldemokratische Gesellschafesprojekı, das diesem Ausdruck hinterliscig assoziicre ist, nicht nur ins Bewußtscin der juristischen Experten cindringen; es müßte im Hintergrundverständnis von Verwalcungen und legislativen Körpcrschaften Whrzeln schlagen - und damic vcriveisen auf die Verschwisterung der verfaßten demokratischen Willensbildung mit autonomen Öffentichkeiten, die gewiß rechtlich ermöglicht werden müssen, aber nicht im ganzen als Körperschaften verfaßr sein können. Das Rechr kann eine demokratische politische Kultur nicht erzeugen, es bleibr von deren Entgegenkommen abhängig. So verstehe ich dic paradoxe Rede von der politischen Ordnung einer Wirtschaftsgesellschaft als Rechtskulturverfassurig.*

Bei Wicthölter kreisen auch die demokratietheoretischen Überlegungen um die Frage, wie "die Unparteilichkeir von Maßstäben, unter denen jeweilige Partikularitäten zu ihrem Recht kommen", institutionalisiert werden können. Skepsis äußert er gegen den abstrakten Universalismus der Aufklärung und ihrer Prinzipien, die nzu hoch über den uns bescimmenden Partikularitäten" angesicdelı sind. Aber ebenso skeptisch isr Wiethölter gegenüber einem Kontextualismus, der dem Universalismus den Rücken kehrt: „Die linke Rechtscheorie hac sich im Kampl ums Recht wohl dahin entschieden, nicht länger im Namen universeller Vernünftigkeir und regulativer Unparteilichkeic Recht einzuklagen, sondern setzt auf radikalisierte Partikularirät, auf die (auch rechtliche) Souveränität und Autonomie selbstbestimmcer Gruppen. Sie reklamiert damit gleichsam Anerkennung absoluter Minoritäı gegenüber relativer Majorität, im Grunde also einen, in klassischer Rechrscerminologie ausgedrückt, eher völkerrechrlichen als bürgerlich-rechrlichen Status. « ${ }^{\circ}$ Wicthölcer selbst setzt dem Modell des Völkerrechts das des Internationalen Privatrechts entgegen: Ein Fall, der aus der Sicht verschiedener Kontexte verschieden interpretiert wird, muß gleichwohl nach Kollisionsregeln entschieden werden, die für beide Seiten akzepcabel sind.

Ähnliches gilt nun für die policische Willensbildung in pluralistischen und hochindividualisierten Gesellschaften, die so weit fragmentiert sind, daß es nur Mehrheiten von subkulturellen Minderheiten geben kann. Diese Situation zwingr keineswegs zur Preisgabe des Universalismus; allein die Anstrengung der Kontextualisierung wächst in dem Maße, wic die Operation der Verallgemeinerung zu immer abstrakteren Regeln oder Grundsätzen nötigt. Darzut reagiert diz wlinke Rechestheoriea of nur mit einem Reflex ihrer alten - auch damals schon unnöcigen - Fehler. Weil sie falschen kollektivistischen Prämissen angehangen hatte, traut sie nun dem Spiegelbild des Individualismus alles zu. U. Preuß benutzt eine reflexiv gewordene Theoric rationaler Wahl, um den gemeinwohlorientierten Staatsbürger Rousseaus von den Höhen seiner nacionalen Identifikacion auf den Bodcn des bürgerlichen Selbstintercsses aufgeklärter Privatleute zurückzuholen. Auf dem Weg von Marx zurück zu Hobbes scheint Kant auf der Strecke zu bleiben: Aus dem individuellen Nutzenkalkül soll über Präferenzen ziveitcr Ordnung die Sozialvertraglichkeic je meincs Interesses hervorgehen. nDie Befriedigung meines individuellen Intercsses hängt also entweder von einer kollektiven Aktion ab - an jeder Ecke stehr ein Polizist, der das Wegwerfverhalten der Passanten beobachect und gegebenenfalls sankcioniert - 
oder davon, daß die anderen Individuen ihr individuelles Verhalten befriedigen, das im Vertrauen auf das gleichgerichtete Verhalten ihrer Mitbürger dann carsächlich

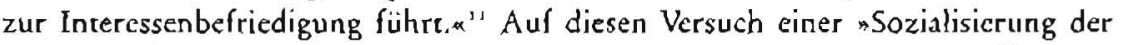
ökonomischen Theorie" hat Wiethölter nicht ganz cindeurig reagiest: »Alles, was Herr Preuß ausfïhrt, ..., erhellt den Kopf ungemein, stiftet aber im Herzen so viel Traurigkcit.*

Nun, ganz so traurig härte Wiethölter bei dieser Gelegenheit nicht sein müssen. In seinem eigenen Diskussionsbeitrag äußerc cr schon einen gesunden Zweifel am individualistischen $Z$ wcifel. Preuß kann nämlich die Kluft zwischen egozencrischem Nutzenkalkül und gemeinsamen Interesse nichr durch Präferenzen schließen, dic auch dann, wenn sie reflexiv werden, subjektiv bleiben. Das leister nur Adam Smith' "unsichtbare Hand* (an die Preuß nicht mehr glaubr) oder Kants nallgeneines Gesetza (eine Geserzgebung, die freilich aus dem Gefängnis des einsamen Seclenlebens befreit werden muß). Preuß selbst sagt es: "Wir haben hier also den Fall nicht einer kollektiven, aber auch nicht einer individuellen, sondern einer auf Reziprozität und wechselseitigem Verrrauen der Individuen beruhenden Interessenbefricdigung. ${ }^{\prime 2} \mathrm{Er}$ postuliert, daß jeder für sich (a) überlegt, welches verallgemeinerte Verhalten für alle gleıchermaßen gut wäre, und (b) darauf vertraut, daß alle andcren dieselbe Überlegung anstellen und (c) nach deren Resultat auch handeln. So springt denn aus dem Hobbes doch wieder der Kant heraus.

Vieliciche solle man von hier aus über Marx zu Mead voranschreiten und mit Wiechölter "Foren und Verfahren* postulieren, die der in foro incerno vollzogenen allgemeinen Perspektivenübcrnahme (ohne die auch die ökonomische Theoric des Rechts nicht "sozialisiert" würde) die feste Gestalt einer intersubjektiven Praxis verlcihen könnten.

II U. PreuB. Perspelesiven von Rechressinal und Demokratie, KV 1, 1989, 10. 